\title{
The Neutron Star Inner Crust: Nuclear Physics Input
}

\author{
Andrew W. Steiner ${ }^{1}$ \\ ${ }^{1}$ Joint Institute for Nuclear Astrophysics, National Superconducting Cyclotron Laboratory, \\ and the Department of Physics and Astronomy, Michigan State University, East Lansing, MI 48824
}

A fully self-consistent model of the neutron star inner crust based upon models of the nucleonic equation of state at zero temperature is constructed. The results nearly match those of previous calculations of the inner crust given the same input equation of state. The extent to which the uncertainties in the symmetry energy, the compressibility, and the equation of state of low-density neutron matter affect the composition of the crust are examined. The composition and pressure of the crust is sensitive to the description of low-density neutron matter and the nuclear symmetry energy, and the latter dependence is non-monotonic, giving larger nuclei for moderate symmetry energies and smaller nuclei for more extreme symmetry energies. Future nuclear experiments may help constrain the crust and future astrophysical observations may constrain the nuclear physics input.

PACS numbers: 26.60.-c,97.60.Jd,21.65.-f,21.60.-n

\section{INTRODUCTION}

The inner crust of a cold neutron star can be defined as the region between the density where neutrons drip out of nuclei (about $4 \times 10^{11} \mathrm{~g} / \mathrm{cm}^{3}$ ) and the density for the transition to homogeneous nucleonic matter at about half of the nuclear saturation density. This region is sensitive to the nuclear physics input because the nature of the crust is determined by the structure of neutron-rich nuclei and the energetics of the surrounding dripped neutrons. In this work, the dependence of the description of the neutron-rich nuclei and the dripped neutrons on the equation of state (EOS) of homogenous nucleonic matter is examined.

The inner crust is of broad interest because a large variety of astrophysical observations are dependent on and sensitive to the properties of the neutron star crust. One recent motivation is the suggestion that the giant flares in Soft Gamma-Ray repeaters trigger seismic events in the neutron star crust and are sensitive to the shear modulus of the crust crust [1, 2, [3]. The shear modulus, in turn, is sensitive to the composition of the neutron star crust and the relative magnitude of the proton and neutron numbers of the nuclei in the inner crust. The moment of inertia of rotating neutron stars is also sensitive to the inner crust and depends on the transition density between the crust and the core [4]. Neutrino and photon opacities are also sensitive to the properties of the nuclei in the inner crust. For example, neutrino-nucleus scattering, which scales like $A^{2}$, is the most important neutrino process during the lepton-trapped phase of a Type II supernova (see Ref. 5] for a recent review). Finally, the cooling and evolution of neutron star crusts depends on the both the size of the crust [6] and by its transport properties [7, 8], which are both related to the composition. These astrophysical connections motivate the study of the magnitude of the uncertainty of the properties of the inner crust which come from present uncertainties in the nuclear physics inputs.

In this article, several models of the neutron star inner crust are constructed systematically using inputs from with the current experimental information while allowing the range of uncertainty allowed due to the uncertainty in the EOS of homogeneous matter. Of particular importance, is that the symmetry energy is varied in both the description of the nuclei and the description of the neutron matter at the same time. The composition depends on the symmetry energy, but is nearly independent of the compressibility. This means that astrophysical observations which are connected to properties in the crust can constrain the nuclear symmetry energy.

The inner crust is quite sensitive to the EOS of neutron matter at sub-saturation densities. At sufficiently low densities, neutron matter is somewhat well understood because three-body interactions are small, and the twobody neutron-neutron interaction is strongly constrained by the experimentally measured neutron-neutron scattering phase shifts [9] (see also the review in Ref. [10]). Many of the currently available EOSs, however, do not respect this understanding of low-density neutron matter because they are fit to the properties of nuclei which are more sensitive to matter near saturation densities. The neutron matter EOSs used in this work are designed to have a realistic behavior below the saturation density, within the precision required for the description of the crust.

\section{THE MASS MODELS}

While microscopically-based models of the nuclei are of great interest because they can disentagle important effects which are not easily treated in a classical approach, a microscopic approach can also make it more difficult to understand the physical principles which guide the nature of the inner neutron star crust. In addition, it is not clear that a classical approach is significantly less effective at estimating the magnitude of uncertainties originating in the nuclear physics input (it may even be more effective). In any case, since the purpose is only to estimate 
the uncertainties from the nuclear physics input to the EOS, a liquid-drop model quite similar to that described in Refs. 11, 12 is used. More microscopic models for the crust have been developed (see the pioneering work of Ref. [13] and recent efforts in Refs. [14, 15]) and it is expected that these results on the sensitivity to the EOS of homogeneous nucleonic matter will apply to some extent in these models as well.

The liquid-drop model for this work consists of a bulk energy contribution which is determined from the EOS of homogenous nucleonic matter together with surface and Coulomb contributions. This will be compared to the finite-range droplet model described in Ref. [16] and used in Ref. [17].

The binding energy per baryon of a nucleus with proton number $Z$ and atomic number $A$ is given by

$$
\begin{aligned}
B(Z, A) / A= & B_{\text {bulk }}\left(n_{n}, n_{p}\right) / A+\sigma \mathcal{B}\left(n_{n}, n_{p}\right)\left(\frac{36 \pi}{n^{2} A}\right)^{1 / 3} \\
& +\mathcal{C} \varepsilon_{\text {Coulomb }} / n
\end{aligned}
$$

where $n_{n}$ and $n_{p}$ are the average neutron and proton densities inside the nucleus with the given $Z$ and $A$. The binding energy of bulk matter, $B_{\text {bulk }}$ (about $-16 \mathrm{MeV}$ in isospin symmetric matter) is given by

$$
B_{\mathrm{bulk}}=\frac{A}{n}\left[\varepsilon\left(n_{n}, n_{p}\right)-n_{n} m_{n}-n_{p} m_{p}\right]
$$

where $m_{n}$ and $m_{p}$ are the neutron and proton masses (which is taken to be $939 \mathrm{MeV}$ ), $n=n_{n}+n_{p}$ is the average baryon number density in the nucleus, and $\varepsilon\left(n_{n}, n_{p}\right)$ is the energy density of homogeneous matter evaluated at the given neutron and proton density. The expression for $\varepsilon$ may be given by any EOS of homogeneous matter and several different models are employed. Note that the energy of the dripped neutrons which is added later will always be determined with the same EOS as is used to describe the bulk part of the nuclear energy.

The average baryon density will be determined from

$$
n=n_{n}+n_{p}=n_{0}+n_{1} I^{2}
$$

where $I=1-2 Z / A$. The parameter $n_{0}$ is analogous to the saturation density of nuclear matter and is expected to be near $0.16 \mathrm{fm}^{-3}$. The parameter $n_{1}$ subsumes (in a very schematic way) two effects: the decrease in the saturation density with the isospin asymmetry and the increase in the saturation density due to the Coulomb interaction. These effects are both explicitly present in the finite range droplet model (see Eq. 49 of Ref. 17]). The decrease in the saturation density with isospin asymmetry is typically larger and thus $n_{1}$ is always negative in these models.

The individual average neutron and proton number densities are given by

$$
\begin{aligned}
& n_{n}=n(1+\delta) / 2 \\
& n_{p}=n(1-\delta) / 2
\end{aligned}
$$

and the density asymmetry $\delta=1-2 n_{p} /\left(n_{n}+n_{p}\right)$ is given by $\delta=\zeta I$ where $\zeta$ is a constant parameter of the model. Neutron and proton radii ("squared-off" radii, not rootmean-square radii) are given simply by $4 \pi n_{n} R_{n}^{3}=3 N$, and $4 \pi n_{p} R_{p}^{3}=3 Z$. The presence of a neutron skin is determined from $\zeta$. If $\zeta$ is unity, then all nuclei have no neutron skin $\left(R_{n}=R_{p}\right)$, while if $\zeta$ is less than unity, then all nuclei with $N>Z$ will have a neutron skin $\left(R_{n}>R_{p}\right)$.

The surface energy contribution is proportional to the surface tension $\sigma, A^{2 / 3}$ (the surface energy scales as $A^{2 / 3}$ so that the surface energy per baryon scales like $A^{-1 / 3}$ as in Eq. 1), and a unitless function $\mathcal{B}$. Typically this latter function is quadratic in the isospin symmetry

$$
\mathcal{B}\left(n_{n}, n_{p}\right)=1-\sigma_{\delta} \delta^{2}
$$

where $\sigma_{\delta}$ is a positive parameter representing the surface symmetry energy. This is essentially the approach taken in Ref. [16]. For a neutron star inner crust model, this can be modified to ensure that the surface energy vanishes in the limit $\delta \rightarrow 1$ as it must. One possible approach (and the one used here) is that from Ref. [12]

$$
\mathcal{B}\left(n_{n}, n_{p}\right)=\frac{16+b}{\left[1 / x^{3}+b+1 /(1-x)^{3}\right]}
$$

where $x=n_{p} / n$ and $b$ is a simple function of the parameter $\sigma_{\delta}$ and is related through $\sigma_{\delta}=96 \sigma /(b+16)$. This is an approximate scheme for taking into account the isospin properties of the surface energy which may suffice for the present purpose, but note the more the more detailed discussion in Ref. [18]. In particular there is still an unresolved ambiguity associated with how the surface energy is handled as discussed in this reference. The slope of the correlation between the surface symmetry energy, $\sigma_{\delta}$ and the symmetry energy at the saturation density depends on the mass formula used. This model, like all other present models for the neutron star crust, effectively chooses a particular slope for this correlation.

The Coulomb energy density of a 3-dimensional droplet of protons can be written [11, 19] (modulo an overall factor of $\chi$, the volume fraction of matter present in nuclei, which is included later),

$$
\varepsilon_{\text {Coulomb }}=\frac{2 \pi}{5} n_{p}^{2} e^{2} R_{p}^{2}\left(2-3 \chi^{1 / 3}+\chi\right)
$$

where $e^{2}$ is the usual Coulomb coupling $\sim \hbar c / 137$. In the final term in parenthesis, the first term corresponds to the standard Coulomb contribution, the second term corresponds to the "lattice contribution" [20] in the WignerSeitz approximation, and the last term to a further finitesize correction relevant at higher densities when $\chi$ is comparable to unity. Note that this last term is quite important near the crust-core transition and tends to delay the transition to nuclear matter to higher densities. The Coulomb contribution is multiplied by a parameter $\mathcal{C}$ to take into account the fact that the proton density does not fall off sharply at a finite radius and this surface 
diffusiveness maybe dependent on the input symmetry energy. This parameter will always be nearly unity. As noted in Ref. [21], the Wigner-Seitz approximation fails when describing the low-temperature transport properties, but will suffice for describing the composition and the equation of state as done here.

In summary, there are six free parameters in this model (outside of the input equation of state of bulk nuclear matter, which is a kind of parameter in itself) are the surface tension in $\mathrm{MeV} / \mathrm{fm}^{2}, \sigma$, the surface symmetry energy $\sigma_{\delta}$, the correction factor to the Coulomb energy, $\mathcal{C}$, the asymmetry parameter $\zeta$, and the central density parameters, $n_{0}$ and $n_{1}$ which are expressed in units of $\mathrm{fm}^{-3}$. These six parameters will be fit to experimental masses for each input EOS.

\section{THE EQUATIONS OF STATE}

The EOS from Ref. 22] (APR) is used, which was obtained from variational chain summation calculations of the equation of state using a realistic nucleon-nucleon interaction. Also, a "typical" relativstic field-theoretical model is utilized (review in Ref. [23]), NL4 [24] which was fit to nuclei. In order to compare with the model of Ref. [25] the Skyrme [26] model SLy4 [27] is used, and in order to compare with the model from Ref. [28] the Skyrme model SkM* 29] is used.

APR is expected to be particularly good for neutron matter at low densities, because it is directly computed from an interaction which reproduces the two-body nucleon-nucleon phase shifts. The model SLy4 also has a good neutron matter EOS because it was fit to both nuclei and low-density neutron matter. The NL4 and SkM* models were only fit to nuclei and low-density neutron matter are less constrained. SkM* happens to have a neutron matter EOS which is somewhat closer to APR than NL4. Like the SLy4 interaction, relativistic models are also able to reproduce, at some level, the more accurate low-density neutron matter EOS found in APR and SLy4, as was demonstrated by the RAPR model in Ref. [18] and the FSUGold model [30, 31].

In order to examine the importance of having an accurate EOS for low-density neutron matter, the low-density neutron matter EOS of NL4 is modified and compared to the original. Three new models are constructed. The first model, NL4Q, is a modification of NL4 which treats the symmetry energy at low densities to be exactly quadratic. This approximation is quite good at lower densities, and the NL4 crust is nearly indistinguishable from the NL4Q crust. The other models, NL4QN and NL4QN2, are versions of NL4Q which reproduces the neutron matter EOS of APR at densities below a specified density,

$$
E_{\text {neut }}^{\mathrm{NL} 4 \mathrm{QN}}=E_{\text {neut }}^{\mathrm{APR}}+\frac{E_{\text {neut }}^{\mathrm{NL} 4 \mathrm{Q}}-E_{\text {neut }}^{\mathrm{APR}}}{1+e^{\left(n_{t}-n\right) / \nu}}
$$

where $n_{t}$ is $0.08(0.04) \mathrm{fm}^{-3}$ and $\nu$ is $0.0105(0.016)$ $\mathrm{fm}^{-3}$ for model NL4QN (NL4QN2). Both NL4QN and

\begin{tabular}{ccccc} 
Model & $\eta_{1}(\mathrm{MeV})$ & $\eta_{2}(\mathrm{MeV})$ & $E_{\text {sym }}(\mathrm{MeV})$ & $\gamma$ \\
\hline Sch & -0.307 & 0.481 & 31 & 0.9 \\
SchS28 & -0.487 & 0.578 & 28 & 0.9 \\
SchS34 & -0.127 & 0.385 & 34 & 0.9 \\
Sch $\gamma 1$ & -0.0308 & 0.198 & 31 & 0.6 \\
Sch $\gamma 2$ & -0.793 & 0.979 & 31 & 1.1 \\
\hline
\end{tabular}

TABLE I: The values of $\eta_{1}$ and $\eta_{2}$ (c.f. Eq. 9), parameters controlling the neutron matter equation of state in the schematic models and the corresponding values of the symmetry energy at saturation density and the exponent $\gamma$. In the schematic models, $\eta_{0}$ is fixed at 0.5.

NL4QN2 have pressures and neutron chemical potentials which monotonically increase with density. The results below show that these two models give signficantly different results for the inner crust.

Finally, several schematic models of the EOS are constructed so that the effect of the compressibility and the symmetry energy can be examined. The schematic EOS for neutron matter is

$$
\begin{aligned}
E_{\text {neut }} / A= & {\left[1-0.6 k_{F, n}^{0.4}+\eta_{1}\left(\frac{n}{n_{0}}\right)+\eta_{2}\left(\frac{n}{n_{0}}\right)^{2}\right] } \\
& \times \frac{k_{F, n}^{5}}{10 \pi^{2} m_{n}}
\end{aligned}
$$

where $k_{F}=\left(3 \pi^{2} n\right)^{1 / 3}$ is the neutron Fermi momentum and the last term is just the free Fermi gas energy density. The first two terms inside the square brackets are designed to reproduce the expectation from equations of state at low densities obtained from two-body potentials which reproduce the experimental phase-shift data on neutron-neutron scattering [9, 32]. It is expected that the interacting neutron matter EOS is about half the free Fermi gas energy at $k_{F, n}=0.5 \mathrm{fm}^{-1}$. The term proportional to $k_{F, n}^{0.4}$, qualitatively reproduces this low-density behavior and the other parameters $\eta_{1}$ and $\eta_{2}$ can be adjusted for densities near the saturation density where the EOS is more uncertain.

It is useful to connect this description with the more traditional description neutron matter in terms of a symmetry energy with the form $E_{\text {sym }}=A\left(n / n_{0}\right)^{2 / 3}+$ $B\left(n / n_{0}\right)^{\gamma}$. For an effective mass of about $0.7 \mathrm{M}, A$ is about $17 \mathrm{MeV}$, and then $B$ and $\gamma$ dictate the magnitude of the symmetry energy at the saturation density and the density dependence of the symmetry energy, respectively. The base model schematic model, "Sch", has values of $\eta_{1}$ and $\eta_{2}$ appropriate for $B=14 \mathrm{MeV}$ (a symmetry energy of $31 \mathrm{MeV}$ ) and $\gamma=0.9$. These values are given in Table I, as well as the values of $\eta_{1}$ and $\eta_{2}$ for the schematic models whose symmetry energy is different from the baseline model. The variation of the value of the symmetry energy at saturation density between 28 and $34 \mathrm{MeV}$ is consistent with the observation that most modern equations of state fall within this range. When the symmetry energy is taken to be a pure power law (the $\mathrm{A}=0$ limit), 
the limit of the variation of $\gamma(0.6<\gamma<1.1)$ is inferred from the experimental information from intermediateenergy heavy-ion collisions [33, 34]. The models Sch $\gamma 1$ and $\operatorname{Sch} \gamma 2$ are constructed by fitting a symmetry energy with the given exponent with $\mathrm{A}=0$ to express the expected range. The extraction of values of $\gamma$ from heavyion collisions is non-trivial, and there may be systematic uncertainties that are not yet understood. These uncertainties would mean that the range of variation presented here is overly conservative, and that the true range might be larger.

The schematic equation of state for nuclear matter is

$$
\begin{aligned}
E_{\text {nuc }} / A= & M+B+\frac{K}{18 n_{0}^{2}}\left(n-n_{0}\right)^{2} \\
& +\frac{K^{\prime}}{162 n_{0}^{3}}\left(n-n_{0}\right)^{3}
\end{aligned}
$$

where $M$ is the nucleon mass, $B$ is the binding energy, $n_{0}$ is the saturation density, $K$ is the compressibility and $K^{\prime}$ is the "skewness". Isospin asymmetric matter is computed assuming that the symmetry energy is exactly quadratic in the isospin asymmetry $\delta$ (this approximation may fail at high density, see Ref. 35]). Note that varying the compressibility in this model is not precisely equal to varying the quantity which might be obtained from giant resonances, as the latter are only sensitive to the equation of state in the neighborhood of saturation density whereas the compressibility is applied to nuclear matter at all densities below the saturation density, thus the variation in the compressibility is a bit larger than that recently suggested in Refs. [36, 37, 38]. The baseline schmatic model "Sch", has a binding energy of -16 $\mathrm{MeV}$, a saturation density of $0.16 \mathrm{fm}^{-3}$, a symmetry energy of $31 \mathrm{MeV}$, a compressibility of $230 \mathrm{MeV}$. In all of the models, the skewness parameter is fixed by ensuring that the energy per baryon of nuclear matter vanishes at zero density, as it ought. In addition to variations of the symmetry energy as discussed above, Two models "SchK210" and "SchK250", are constructed to be the same as the baseline model, except that they have different compressibilities.

A survey of some of the equations of state is given in Fig. 1. The upper right panel shows how neutron matter in the NL4QN and NL4QN2 models interpolates between APR at low densities and the normal NL4Q model at higher densities, while leaving the nuclear matter EOS unmodified.

\section{THE MASS FITS}

The liquid drop model is fit to the experimental nuclear masses from Ref. [39] using the fitting formula

$$
\Delta_{\mathrm{RMS}}=\left[\frac{1}{N} \sum_{i=1}^{N}\left(M_{i}^{e x p}-M_{i}^{t h}\right)^{2}\right]^{1 / 2}
$$

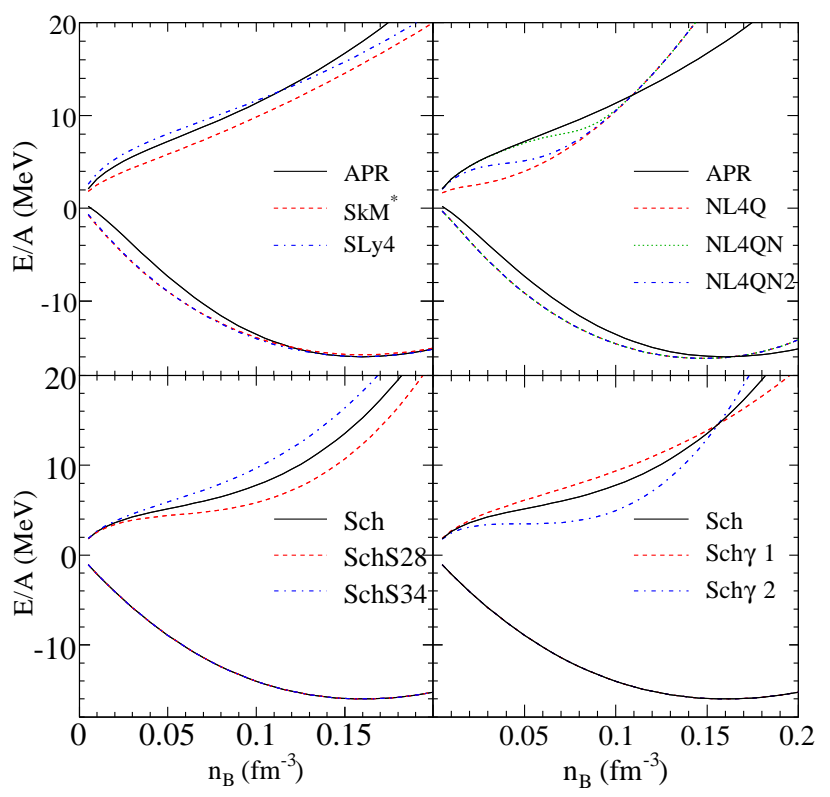

FIG. 1: A survey of the equations of state used in this work. Plotted in each panel are the energy per baryon of nuclear matter (lower set of curves) and neutron matter (upper set of curves) as a function of baryon density, $n_{B}$. The upper left panel shows the models APR, SLy4, and SkM*, the upper right panel shows the model NL4Q (based on NL4) and shows how neutron matter was modified to match APR at low densities, and the lower panels show how the symmetry energy was modified in the schematic equation of state.

\begin{tabular}{cccccccc} 
Model & $\zeta$ & $\sigma$ & $\sigma_{\delta}$ & $\mathcal{C}$ & $n_{1}$ & $n_{0}$ & $\Delta_{\mathrm{RMS}}$ \\
\hline APR & 0.886 & 1.19 & 1.72 & 0.885 & -0.128 & 0.181 & 2.61 \\
SkM $^{*}$ & 0.888 & 1.14 & 1.16 & 0.899 & -0.0612 & 0.17 & 2.61 \\
SLy4 & 0.885 & 1.19 & 1.57 & 0.882 & -0.11 & 0.181 & 2.6 \\
NL4Q & 0.89 & 1.15 & 2.67 & 0.915 & -0.234 & 0.169 & 2.66 \\
Sch & 0.897 & 1.19 & 1.67 & 0.903 & -0.12 & 0.176 & 2.7 \\
SchK210 & 0.897 & 1.19 & 1.69 & 0.907 & -0.117 & 0.174 & 2.72 \\
SchK250 & 0.897 & 1.19 & 1.64 & 0.9 & -0.12 & 0.176 & 2.69 \\
SchS28 & 0.891 & 1.2 & 1.18 & 0.892 & -0.0488 & 0.179 & 2.66 \\
SchS34 & 0.9 & 1.19 & 2.44 & 0.909 & -0.206 & 0.175 & 2.68 \\
Sch $\gamma 1$ & 0.891 & 1.2 & 1.49 & 0.884 & -0.103 & 0.182 & 2.63 \\
Sch $\gamma 2$ & 0.911 & 1.11 & 0.978 & 0.954 & -0.028 & 0.154 & 2.75 \\
\hline
\end{tabular}

TABLE II: The nuclear mass fits corresponding to the models described in the text. The values of $\sigma$ are given in $\mathrm{MeV} / \mathrm{fm}^{2}$ and $n_{0}$ and $n_{1}$ are given in $\mathrm{fm}^{-3}$.

where $N$ is the number of nuclei, and $M^{\exp }$ and $M^{\text {th }}$ are the experimental and theoretical values of the mass excess. Ref. 17] points out that this fitting formula can be improved and that it overestimates the actual model error, but these considerations will not be important at the level of the results presented here. The fitting results are given in Table II. The fitting results for NL4QN and NL4QN2 are not given because they were found to be nearly equal to those from NL4Q. 
The mass fit is performed by minimizing $\Delta_{\mathrm{RMS}}$ for all the experimentally measured mass excesses from Ref. [39]. For each nucleus, this involves computing the neutron and proton densities using Eqs. 3 and 4 computing the bulk energy from the EOS of homoegenous matter at these densities, then inserting this bulk energy into the nuclear mass formula to compute the mass excess using Eq. 10

As expected, there is a correlation between the surface symmetry energy and the symmetry energy as shown by the increase in $\sigma_{\delta}$ when going from model SchS28 to model SchS34. This is also the reason why NL4 gives a larger value of $\sigma_{\delta}$ than the other models. The value of $\sigma_{\delta}$ is nearly unchanged by modifying $\gamma$, which changes the density dependence of the symmetry. The values of $n_{0}$ in Table IV are not quite equal to the saturation density for homogeneous nuclear matter, and this can be attributed to finite-size effects not captured in Eq. 3. The other parameters are nearly unchanged between models except for $n_{1}$ which is also sensitive to the symmetry energy, as well as the Coulomb interaction.

\section{THE CRUSTS}

In order to determine the composition and properties of the crust, the energy at a fixed density as a function of the proton number and atomic number of nuclei, and the number density of dripped neutrons, $n_{n, \text { drip }}$, is minimized. The energy of matter in the neutron star crust is given by

$$
\begin{aligned}
\varepsilon\left(Z, A, n_{n, \text { drip }}\right)= & \left(n_{n}+n_{p}\right) \chi B(Z, A) / A+ \\
& (1-\chi) \varepsilon_{\text {drip }}\left(n_{n, \text { drip }}\right)+\varepsilon_{\text {el }}\left(n_{e}\right)(12
\end{aligned}
$$

This energy is minimized over the three parameters $Z, A$, and $n_{n \text {,drip }}$ at each density. The volume fraction of matter inside nuclei, $\chi$, is determined from the relation

$$
n_{B}=\chi\left(n_{n}+n_{p}\right)+n_{n, \text { drip }}(1-\chi)
$$

The inner crust implied by the models NL4Q, NL4QN and NL4QN2 are compared in Fig. 2, While the actual number density of dripped neutrons is not strongly modified by modifying the neutron matter EOS, the nuclear size is modified by $50 \%$ or more. The larger energy cost of creating neutron matter in with a more realistic neutron matter EOS is reflected in moving neutrons into nuclei so as not to pay the energy cost. Because the largest difference is between the models NL4Q and NL4QN2, it is clear that most of the dependence on the low-density neutron matter EOS lies at densities below $0.04 \mathrm{fm}^{-3}$.

In order to show the effect due to changing the mass model, Fig. 3 shows the results for the APR EOS with the two different models, the liquid drop model and the FRDM. The results are qualitatively the same but quantitatively different. The form of the mass model remains a significant uncertainty in the nature of the neutron star

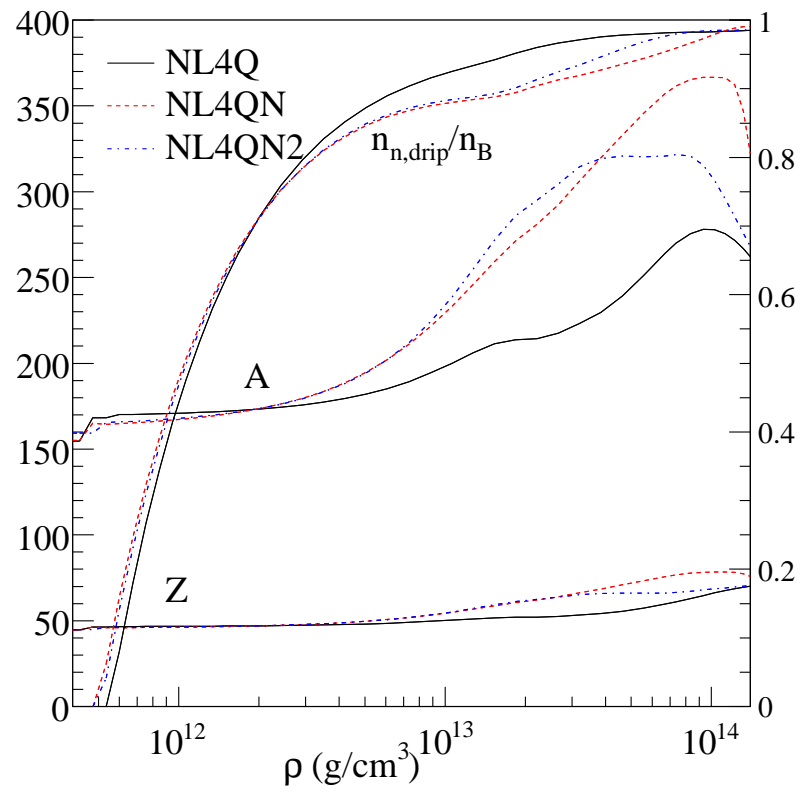

FIG. 2: A comparison of the composition of the neutron star crusts in models NL4Q, NL4QN, and NL4QN2, designed to demonstrate the sensitivity of the composition to the equation of state of low-density neutron matter. The proton nuber, Z, the atomic number A (left axis) and the number density of the dripped neutrons $n_{\mathrm{n} \text {,drip }}$ are displayed (right axis).

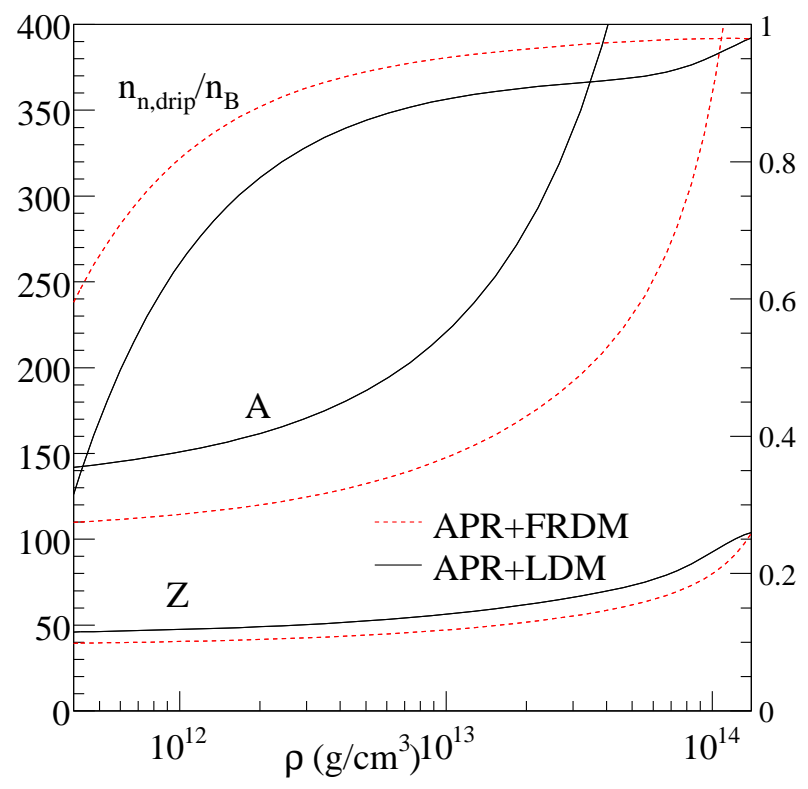

FIG. 3: A comparison the composition given the two different mass models. The curve labeled LDM (solid lines) is obtained from Eq. 1. and the curve labeled FRDM (dashed lines) is obtained from Ref. [17]. The left axis is for $A$ and $Z$ and the right axis is for $n_{\mathrm{n} \text {,drip }}$. 


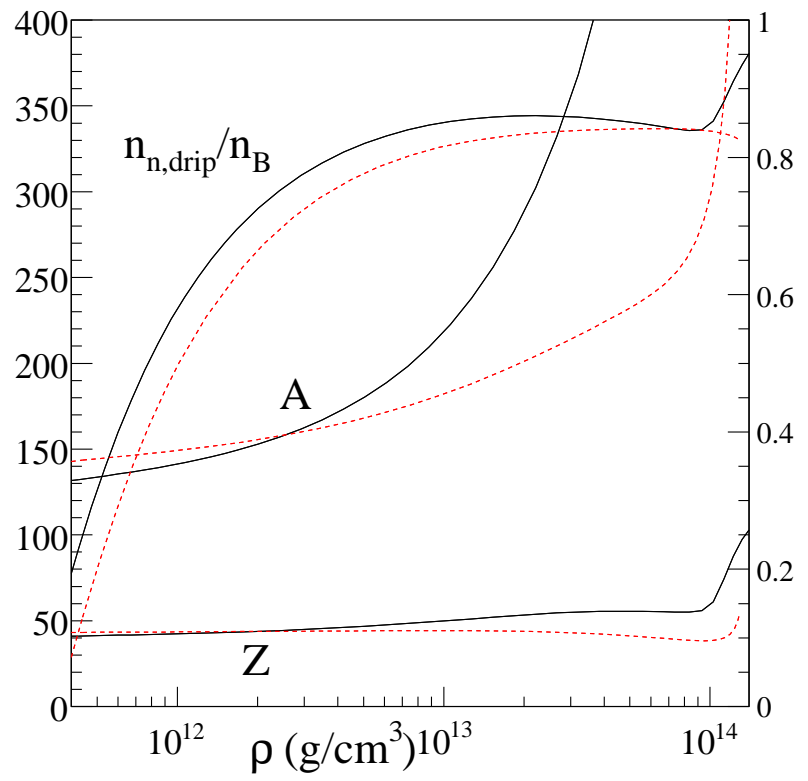

FIG. 4: A comparison of the present work (solid lines) with the results of Ref. [25] (dashed lines) using the same input equation of state, SLy4. The left axis is for $A$ and $Z$ and the right axis is for $n_{\mathrm{n}, \mathrm{drip}}$.

crust, and is comparable to the other uncertainty obtained from the symmetry energy as described below.

Comparisons of the present model to that of Refs. 25, 28] are given in Figs. 4 and 5. The same input EOS for homogeneous matter as the original reference is used in both cases. The results agree qualitatively with the aforementioned works. The remaining differences lie within the nuclear mass formula used, and they are within the range of variation which is suggested by Fig. 3. This model (like all other models of the neutron star crust presently available) cannot precisely predict the composition of the inner crust. Nevertheless, it is qualitatively correct and is thus useful for estimating the uncertainties due to the input EOS of homogeneous matter.

To compare the effect of the uncertainty in the symmetry energy, Fig. 6 shows the composition for the neutron star crust as a function of density for the schematic equations of state with different symmetry energies. The naive expectation is that a stronger symmetry energy tends to encourage nuclei to become more isospinsymmetric. This is coupled, however, with the fact that an increased symmetry energy will also raise the energy cost for the dripped neutrons. These two effects together could force larger, more symmetric nuclei, but this also affects the Coulomb and surface energy contributions. The variation of the composition with the value of the symmetry energy is not so clear, as the baseline model predicts larger nuclei than either models with smaller or larger values of the symmetry energy.

In order to disentangle this result, more detailed results for schematic models with different symmetry energies

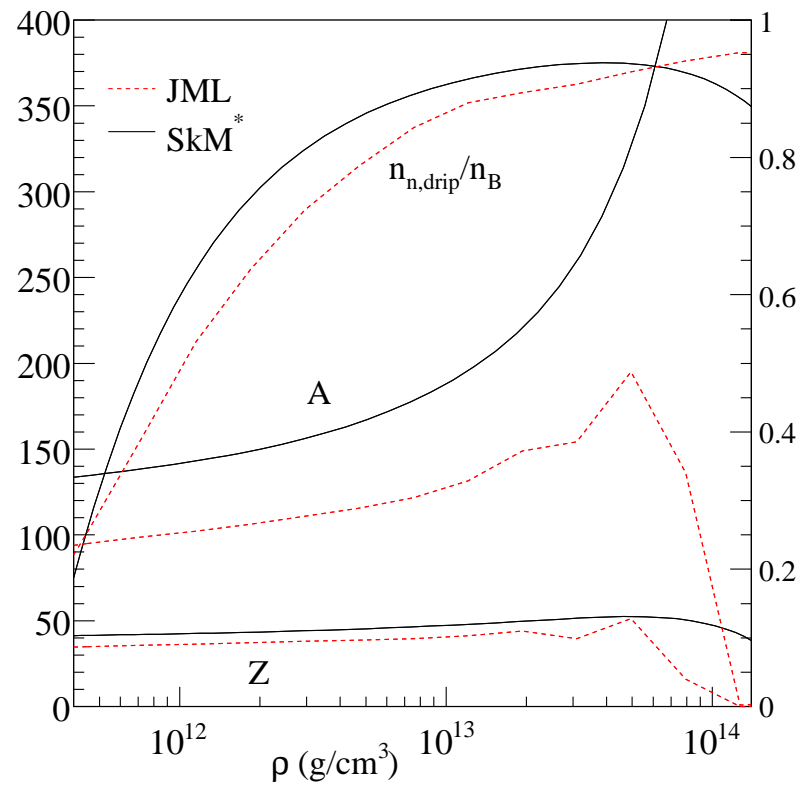

FIG. 5: A comparison of the present work (solid lines) with the results from Ref. 28] (labelled "JML" and plotted with dashed lines) using the same input equation of state, $\mathrm{Sk} M^{*}$. The sharp dropoff in JML at high-densities is due to the transition to homogeneous nucleonic matter. The slightly jagged nature of the JML results is due to the naive interpolation employed in this work and is not necessarily present in the original table. The left axis is for $A$ and $Z$ and the right axis is for $n_{\mathrm{n}, \mathrm{drip}}$.

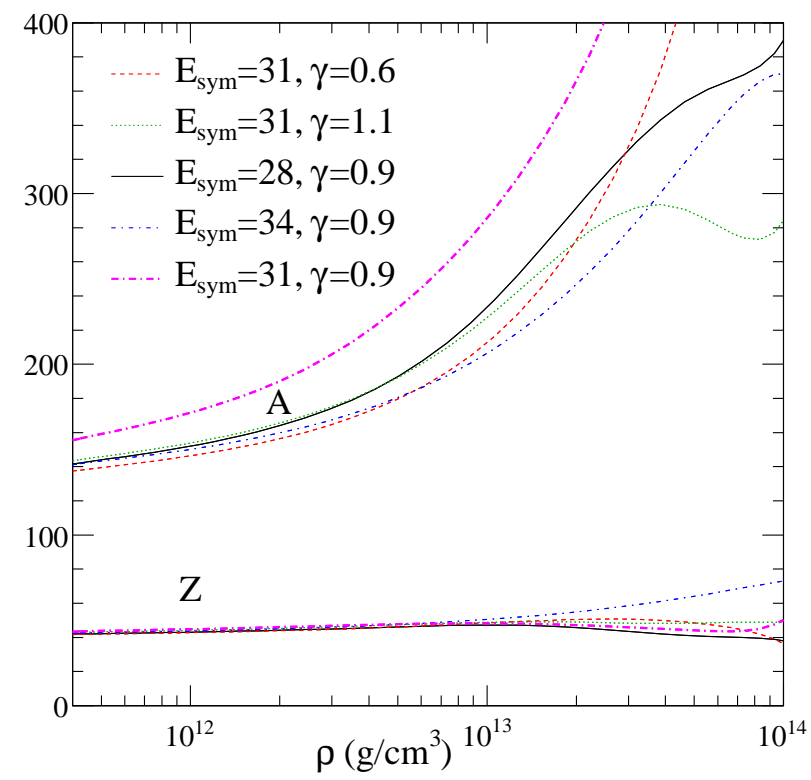

FIG. 6: A comparison of the composition of the crust given different symmetry energies. The bold solid line is the baseline model, the dashed and dotted lines show the variation in $\gamma$, and the dashed-dotted and thin solid line give the variation in the magnitude of the symmetry energy at the saturation density. 


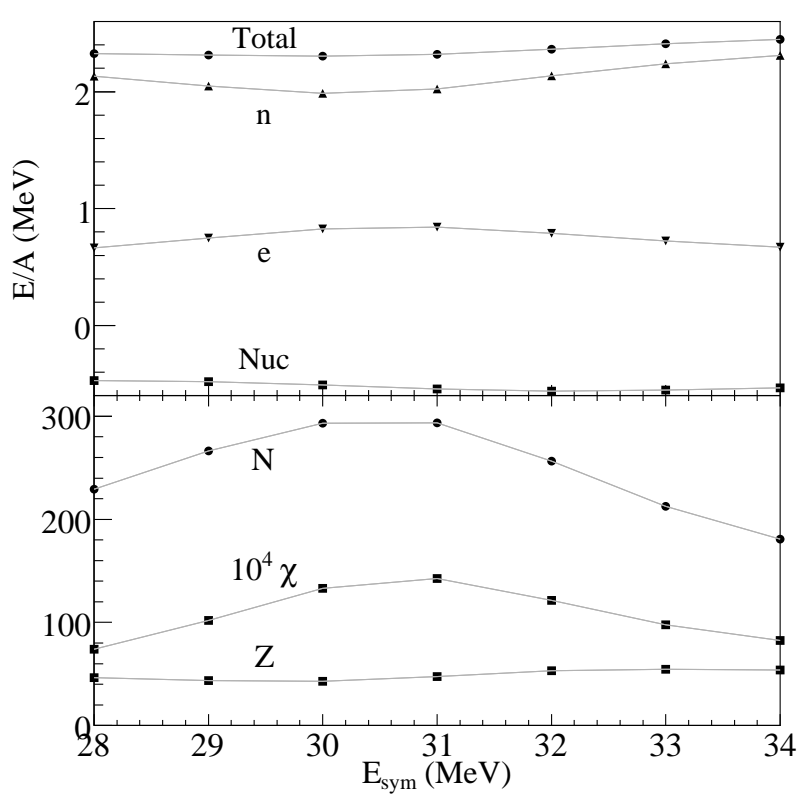

FIG. 7: A comparison of the composition of the crust given different values of the symmetry energy at the saturation density at a fixed density of $n_{B}=0.01 \mathrm{fm}^{-3}$. The top panel gives the total binding energy per baryon, and the separate contributions from dripped neutrons ("n"), electrons ("e"), and nuclei ("Nuc"). The bottom panel shows the neutron and proton number of nuclei as well as the volume fraction, $\chi$.

are given in Fig. [ 7 at a fixed density of $n_{B}=0.01 \mathrm{fm}^{-3}$. Beginning with the larger symmetry energy (with a value at saturation of $34 \mathrm{MeV}$ ) and proceeding downward, the expected result is obtained: lower symmetry energies allow the system to create more isospin-asymmetric nuclei. At low enough symmetry energies, however, this becomes too costly as the electron contribution to the energy increases (the proton number decreases, but the volume fraction occupied by nuclei increases, thus the electron density must increase). Instead, the system reponds by moving neutrons out of the nuclei, which lowers the electron contribution, even though it increases the contributions from nuclei and the dripped neutrons. This is allowed, in part, because the nuclei are able to maintain a relatively constant energy. They can do this because the surface and Coulomb energy cost is cancelled by the bulk energy gain which results from making nuclei with a larger (in absolute magnitude) bulk binding energy.

Finally, Fig. 8 summarizes the pressure as a function of the baryon density. The upper left panel shows the variation from the different mass formulas, which is larger at lower densities. The upper right panel shows the results for $\mathrm{SkM}^{*}$ and SLy4. The lower-left panel shows the variation allowed by the symmetry energy, and variations of up to a factor of two in the pressure are implied by the uncertainty in the symmetry energy. The pressure appears sensitive to the magnitude of the symmetry energy and its dependence on density. Finally, the lower-right

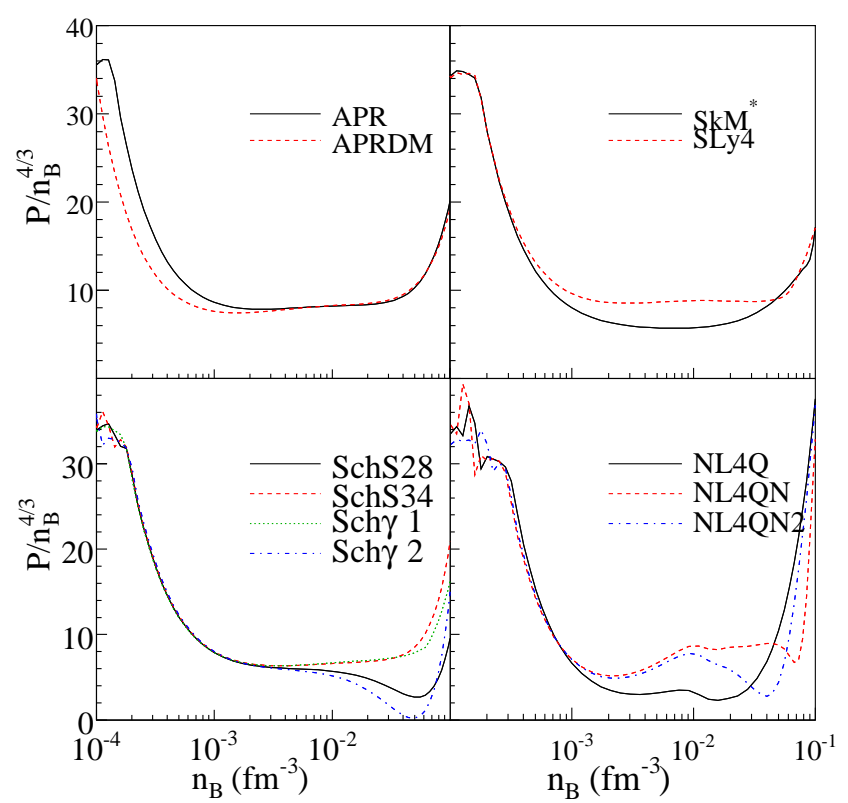

FIG. 8: A survey of the pressure of the crust as a function of density, scaled by the baryon density, $n_{B}^{4 / 3}$. The curves are nearly flat where the adiabatic index is expected to be nearly $4 / 3$ since the baryon density nearly scales with the energy density.

panel shows the pressure for the NL4Q-related models. Note that for model NL4Q, the anomalously small EOS of low-density neutron matter underestimates the pressure in the inner crust. All of the models are connected (sometimes discontinuously) to the EOS from Ref. 20] at low densities thus giving the scatter in the pressure at the lowest densities given in this figure. Particularly interesting is that the pressure at the crust at the higher densities is nearly independent of the mass model (as shown in the upper left panel), which may indicate that astrophysical observables which are sensitive to the pressure of the crust rather than the composition are good probes of the nuclear symmetry energy, assuming that the description of low-density neutron matter is correct.

The transition density to homogeneous nuclear matter is computed by noting the density at which the energy per baryon of nuclear matter becomes smaller than that of the heterogeneous phase. The results for the transition density are given in Table III. The transition densities from this work are given in the second column and third column contains the transition densities for previous works with similar input EOSs for comparison. These transition densities may be underestimates, as the ability of nuclei to deform slightly will decrease the energy of the heterogeneous phase and thus increase the transition density. This will be addressed in further work. Note that, as in the composition discussed above, the transition densities depend non-trivially on the symmetry energy. 


\begin{tabular}{ccc} 
Model & $n_{t} \mathrm{fm}^{-3}$ & \\
\hline APR & 0.0522 & \\
SkM$^{*}$ & 0.0434 & $0.045[28]$ \\
SLy4 & 0.0669 & $0.076[25]$ \\
NL4Q & 0.0344 & \\
NL4QN & 0.0409 & \\
NL4QN2 & 0.0333 & \\
Sch & 0.0584 & \\
SchK210 & 0.0585 & \\
SchK250 & 0.0591 & \\
SchS28 & 0.0368 & \\
SchS34 & 0.0416 & \\
Sch $\gamma 1$ & 0.0676 & \\
Sch $\gamma 2$ & 0.0641 & \\
\hline
\end{tabular}

TABLE III: Caption here.

\section{CONCLUSIONS}

The composition of the neutron star crust is still partially unknown, due to uncertainties in the nuclear mass formula and the equation of state. The composition (and to a lesser extent, the overall pressure) is quite sensitive to the equation of state of low-density neutron matter, and the nuclear symmetry energy, both its magnitude and its density dependence. The dependence of the composition on the symmetry energy is not monotonic, as models with moderate symmetry energies can have larger nuclei than models with lower or higher symmetry energies. To the extent to which neutron stars depend on the composition, this means that it is important to explore the full range of variation in the crust allowed by the present knowledge of the input nuclear physics, while ensuring that the EOS is constrained by what is already known about the EOS of low-density neutron matter. Nuclear experiments will continue to provide better constraints on the symmetry energy, including from the PREX experiment [40, 41] to measure the neutron skin thickness of lead at Jefferson Lab and from intermediateenergy heavy-ion collisions as has been done in Ref. [33.

It remains to be seen if these results persist in the more microscopic models which include pairing, corrections beyond the Wigner-Seitz approximation, long-range correlations, and better treatments of the nuclear structure.

\section{ACKNOWLEDGEMENTS}

The author would like to thank Ed Brown, Joe Carlson, Alex Gezerlis, Jim Lattimer, Bill Lynch, Sanjay Reddy, Sergio Souza, and Anna Watts for useful discussions related to this work. This work is supported by Joint Institute for Nuclear Astrophysics at MSU under NSF-PFC grant PHY 02-16783.
[1] C. Thompson and R. C. Duncan, Mon. Not. Roy. Astron. Soc. 275, 255 (1995).

[2] T. E. Strohmayer and A. L. Watts, Astrophys. J. 653, 593 (2006).

[3] L. Samuelsson and N. Andersson, Mon. Not. Roy. Astron. Soc. 374, 256 (2007).

[4] B. Link, R. I. Epstein, and J. M. Lattimer, Phys. Rev. Lett. 83, 3362 (1999).

[5] A. Burrows, S. Reddy, and T. A. Thompson, Nucl. Phys. A 777, 356 (2006).

[6] J. M. Lattimer, K. A. van Riper, M. Prakash, and M. Prakash, Astrophys. J. 425, 802 (1994).

[7] A. Y. Potekhin, G. Chabrier, and D. G. Yakovlev, Astron. Astrophys. 323, 415 (1997).

[8] E. F. Brown, Astrophys. J. 531, 988 (2000).

[9] J. Carlson, J. Morales Jr., V. R. Pandharipande, and D. G. Ravenhall, Phys. Rev. C 68, 025802 (2003).

[10] H. Heiselberg and V. R. Pandharipande, Ann. Rev. Nucl. Part. Sci. 50, 481 (2000).

[11] G. Baym, H. A. Bethe, and C. J. Pethick, Nucl. Phys. A 175, 225 (1971).

[12] J. M. Lattimer, C. J. Pethick, D. G. Ravenhall, and D. Q. Lamb, Nucl. Phys. A 432, 646 (1985).

[13] J. W. Negele and D. Vautherin, Nucl. Phys. A 207, 298 (1983).

[14] M. Baldo, E. E. Saperstein, and S. V. Tolokonnikov, Phys. Rev. C 76, 025803 (2007).

[15] W. G. Newton, J. R. Stone, and A. Mezzacappa,
arXiv:0708.3197 (2007).

[16] W. D. Myers and W. J. Swiatecki, Ann. Phys. 55, 395 (1969).

[17] P. Möller, J. R. Nix, W. D. Myers, and W. J. Swiatecki, At. Data and Nucl. Data Tables 59, 185 (1995).

[18] A. W. Steiner, M. Prakash, J. M. Lattimer, and P. J. Ellis, Phys. Rep. 411, 325 (2005).

[19] D. G. Ravenhall, C. J. Pethick, and J. R. Wilson, Phys. Rev. Lett. 50, 2066 (1983).

[20] G. Baym, C. Pethick, and P. Sutherland, Astrophys. J. 170, 299 (1971).

[21] N. Chamel, S. Naimi, E. Khan, and J. Margueron, Phys. Rev. C 75, 055806 (2007).

[22] A. Akmal, V. R. Pandharipande, and D. G. Ravenhall, Phys. Rev. C 58, 1804 (1998).

[23] B. D. Serot and J. D. Walecka, in Adv. Nucl. Phys., edited by J. W. Negele and E. Vogt (Plenum, 1989), vol. 16, p. 1.

[24] B. Nerlo-Pomorska and J. Sykut, Int. J. Mod. Phys. E 13, 75 (2004).

[25] F. Douchin and P. Haensel, Astron. and Astrophys. 380, 151 (2001).

[26] T. H. R. Skyrme, Nucl. Phys. 9, 615 (1959).

[27] E. Chabanat, thesis (unpublished) (1995).

[28] J. M. Lattimer, Tables for model SkM* obtained from www.astro.sunysb.edu/lattimer/EOS/main.html, (These are updates of the previous work in 42]).

[29] J. Bartel, P. Quentin, M. Brack, C. Guet, and H.-B. Høakansson, Nucl. Phys. A 386, 79 (1982). 
[30] B. G. Todd-Rutel and J. Piekarewicz, Phys. Rev. Lett. 95, 122501 (2005).

[31] J. Piekarewicz, arXiv:0709.2699 (2007).

[32] A. Gezerlis and J. Carlson, (to be submitted) (2007).

[33] M. B. Tsang, T. X. Liu, L. Shi, P. Danielewicz, C. K. Gelbke, X. D. Liu, W. G. Lynch, W. P. Tan, G. Verde, A. Wagner, et al., Phys. Rev. Lett. 92, 062701 (2004).

[34] L.-W. Chen, C. M. Ko, and B.-A. Li, Phys. Rev. Lett. 94, 032701 (2005).

[35] A. W. Steiner, Phys. Rev. C 74, 045808 (2006).

[36] B. K. Agrawal, S. Shlomo, and V. K. Au, Phys. Rev. C 68, 031304 (2003).
[37] G. Coló, N. Van Giai, J. Meyer, K. Bennaceur, and P. Bonche, Phys. Rev. C 70, 024307 (2004).

[38] U. Garg, nucl-ex/0608007 (2006).

[39] G. Audi, A. H. Wapstra, and C. Thibault, Nucl. Phys. A 729, 337 (2003).

[40] R. Michaels, P. A. Souder, and G. M. Urciuoli, Jefferson Laboratory Proposal PR-00-003 (2000).

[41] C. J. Horowitz, S. J. Pollock, P. A. Souder, and R. Michaels, Phys. Rev. C 63, 025501 (2001).

[42] J. M. Lattimer and F. D. Swesty, Nucl. Phys. A 535, 331 (1991). 